\title{
Scientific results with INTEGRAL
}

\section{Th. Courvoisier}

ISDC Science Data Centre, Versoix, Switzerland

thierry.courvoisier@obs.unige.ch

\begin{abstract}
.
Instruments will be briefly introduced and the main mission results to date will be described.
\end{abstract}

\section{Discussion}

MiRABEL: Has any spatial asymmetry or time variability been detected in the $511 \mathrm{keV}$ emmission from the Galactic Centre region?

Courvoisier: No, the emission is symmetrical (in the limits of the statistics) and has not been seen to be variable. The latter is expected from an extended source.

ELVIS: Is there any clue as to where the heavy obscuration toward the "Type 2" X-ray binaries lies?

Courvoisier: Yes, the material hiding the X-ray source is local to the emitting system and such that the companion is not obscured. 